\title{
The Simulation and Analysis of the Tilt Rotor Aircraft Model Transition
}

\author{
Min $\mathrm{Ji}^{1, \text { a }}$, Ning Wang ${ }^{2, \mathrm{~b}}$ and Chong Zhao ${ }^{3, \mathrm{c}}$ \\ ${ }^{1}$ Xijing University, Shaanxi Xi'an, 710123, China \\ a995010771@qq.com, b15905721@qq.com, c34680572@qq.com
}

Keywords: Simulation; XV-15; Tilt rotor

\begin{abstract}
Based on the results of simulation, operating characteristics of $\mathrm{XV}-15$ tilt rotor aircraft were qualitative analyzed, and when to fade out the rotor control was determined, and also the conversion corridor was determined. Inside the conversion corridor, a conversion route that had the least trimmed pitch attitude was suggested.
\end{abstract}

\section{Introduction}

There are two kinds of tilt rotor aircraft work mode, helicopter mode and the fixed wing machine. This paper mainly on the transition between the two kinds of working mode is analyzed, when these two kinds of working mode to switch from the transition, the most important thing is to determine a safe transition corridor, namely in different nacelle tilting Angle can reach the speed range.

\section{The Transition the Theoretical Research of the Corridor}

Tilt rotor aircraft is both a helicopter model, and have a fixed wing machine model. Between model of helicopter and fixed wing machine the range of the speed limit in the different inclination Angle by the transitional corridor said.

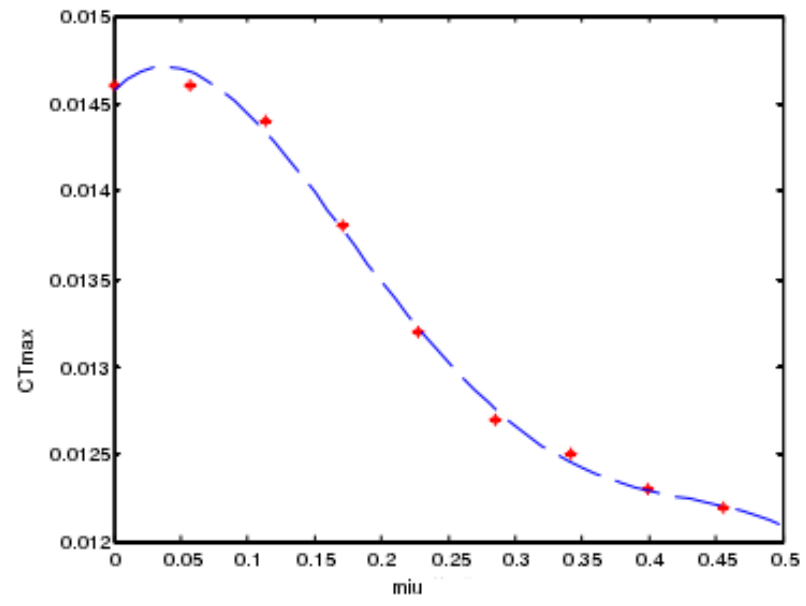

Figure 1. rotor maximum tension coefficient and forward than relationship curve

Between the model of helicopter and fixed wing machine transition, airplanes are caused by the airfoil stall on the one hand lift flash small plane was caused by the lift can't support the weight of the whole plane, on the other hand also because rotor blade stall and go on after shock wave produced by the blade and make the rotor thrust produced by the limited, leading to rotor and wing, and other lifting surface produced by $\mathrm{z}$ negative force can't support the weight of the whole plane.Fig. 1 for rotor maximum tension coefficient and forward than relationship curve. In transition section, therefore, not only the minimum speed limit, and maximum flying speed limit.

\section{The Transitional Period of Simulation Research}

Transition corridor simulation research is focused on the study on balancing the tilt rotor aircraft. This article through to the XV -15 balancing the simulation analysis of tilt rotor aircraft, the model 
of helicopters, fixed-wing and control characteristics were analyzed, and the transition section and carried out simulation analysis on the manipulation of a transition section, when to exit the rotor the advice of the manipulated variable is given.

In this paper mainly analysis model of helicopters, fixed-wing and nacelle tilting Angle in $15^{\circ}$, $30^{\circ}, 45^{\circ}, 60^{\circ}, 75^{\circ}$ balancing control characteristics. Before giving characteristic need clear, mathematical model in this paper, the total is apart from the Angle of theta $\theta 0$ is contains $40^{\circ}$ twisted blade root.

The Helicopter Mode. In nacelle Angle $\beta M=0$ helicopter mode, for balancing XV-15.As shown in Fig.2, is given in the helicopter mode to control the attitude with usual XV to 15 input as the change of speed increase trend.

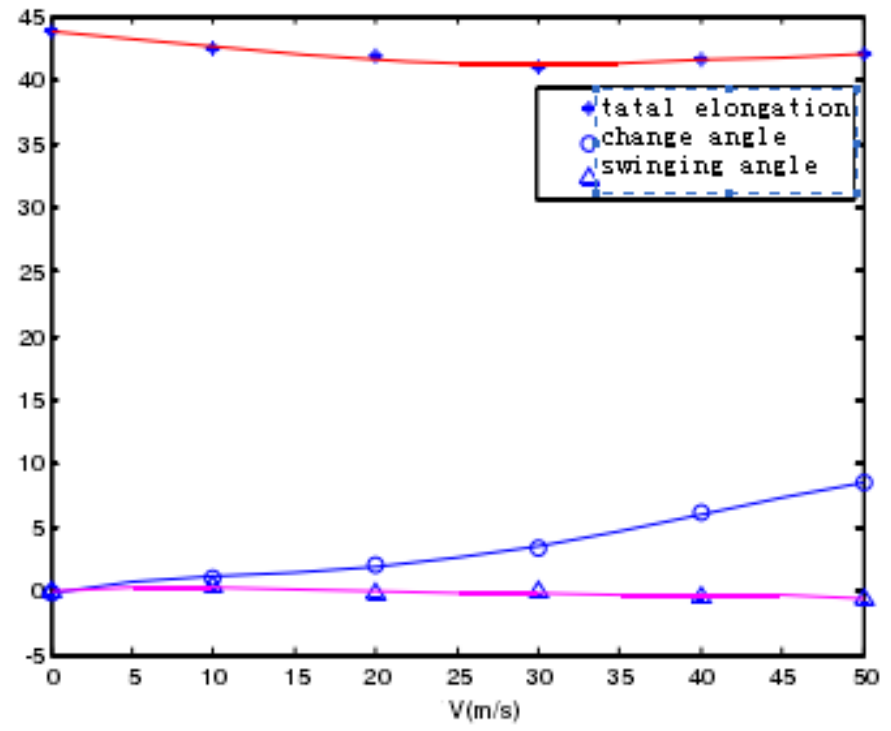

Figure 2. input along with the speed change trend

The helicopter model hovering state, rotor wake aerodynamic interference is more serious on the wing, wing slip flow area on the surface of the area in the maximum in the transition process. Plane at this time of the whole lift mainly provided by the rotor, and because of the influence of the rotor wake even down the wing of the force, which requires the rotor generate greater thrust to bear the weight of the whole plane, and thus in a hover state when the plane from the total control input is the largest. And elevator while in the process of the flight, but in helicopter mode because of the speed is very small, its efficiency is very low, so in the hover state, elevator deflection Angle is zero.

With the increase of speed, rotor wake aerodynamic interference of wing gradually decreases, and the wing produce lift up gradually, auxiliary rotor bear the weight of the plane, so the rotor total elongation gradually smaller. But when the speed reaches a certain value (about $35 \mathrm{~m} / \mathrm{s}$ ) aircraft resistance increases, so the need to increase the total distance to keep the balance of the fuselage. With the increase of the speed, longitudinal cycle variable pitch also needs increasing to keep the balance of the body in the helicopter mode, elevator deflection angle of balancing. Angle has been very small, so in helicopter mode can consider to use the elevator deflection Angle to balancing.

Transition Section. Each during the transition period of the nacelle tilting Angle of $15^{\circ}, 30^{\circ}, 45^{\circ}$, $60^{\circ}, 75^{\circ}$ for balancing planes. Fig. 3 shows the transition period of balancing the total distance as increases with the degree of change trend. 


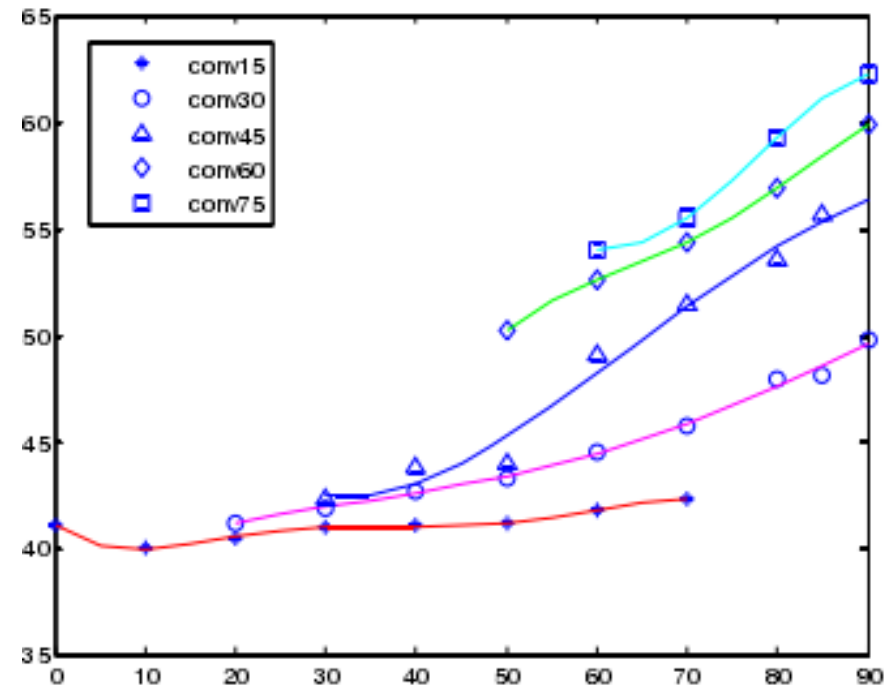

Figure 3. transition section balancing the total distance change with speed

As can be seen from the Fig. 3, with the increase of speed, Angle of nacelle, with the increasing of the total distance is more and more big. In nacelle Angle is $15^{\circ}$, total elongation trend along with the change of velocity is similar to the helicopter model, total elongation increase with the decrease of the first. This is mainly because the nacelle Angle in $15^{\circ}$, main lift or provided by the rotor, along with the rising of the forward speed, rotor wake aerodynamic interference of wing gradually decreases, and provide the wing of the lift force increases gradually, the amount of total distance required rotor can be slightly reduced. And with the speed increasing, the plane resistance increases, and therefore need to increase the total distance to keep the balance of the fuselage.

Fixed-Wing Machine Model. Fig. 4 shows the fixed-wing mode of machine balancing control input with the speed change trend chart. Fixed-wing machine mode, the nacelle tilting to the wing and the level of position, at this time of the rotor thrust as thrust of the plane, with the increase of the speed of the plane's resistance also gradually increase, so you need to increase the total elongation of the trend to provide a gradual increase in before the plane fly propulsion.

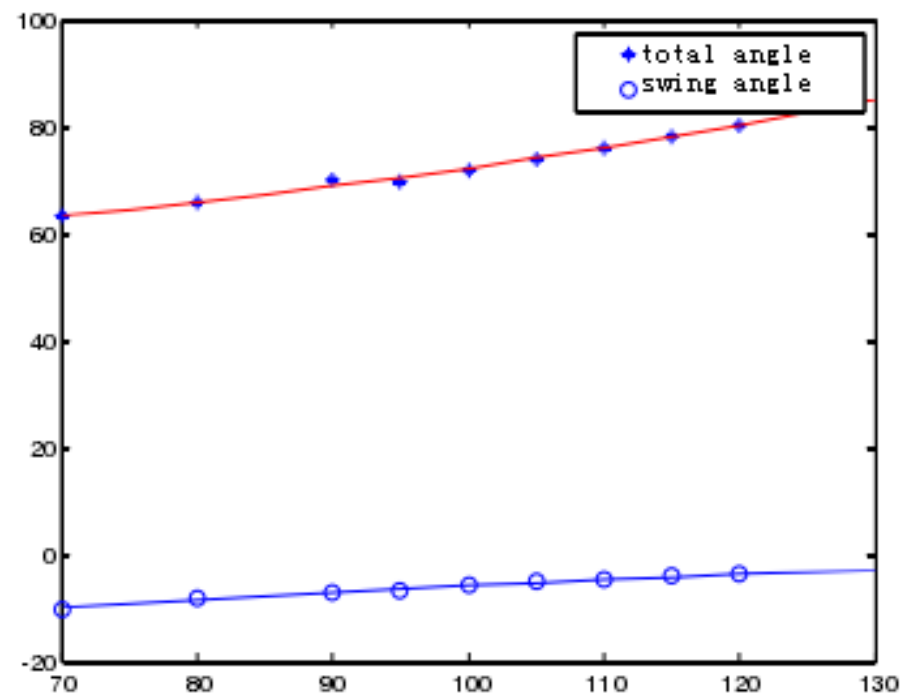

Figure 4. fixed-wing machine model balancing control input change trend

\section{The Transition Simulation Research of the Corridor}

Before the simulation study on the transition of corridor, it is necessary to analyze the XV - 15 balancing process. Aircraft gravity is constant, so the $\mathrm{X}, \mathrm{Y}, \mathrm{Z}$, theta quantity of the three are interrelated, especially $\mathrm{Z}$ to force and pitching Angle theta, if the plane $\mathrm{Z}$ negative force produced 
by all the lifting surface is too small, the inevitable need pitching Angle theta is very big to finish balancing .In this article, therefore, the transition of the corridor requirement is to assume that the scope of the balancing of the pitch Angle after is $20^{\circ} \leqslant \theta \leqslant 20^{\circ}$.In the future in the research on the transition of tilt rotor model aircraft corridor can be to determine the scope of the balancing of the pitching Angle according to the requirement, to assume a range here. Fig. 5 shows the XV when different nacelle Angle speed - balancing the pitch attitude Angle change rule of XV-15.

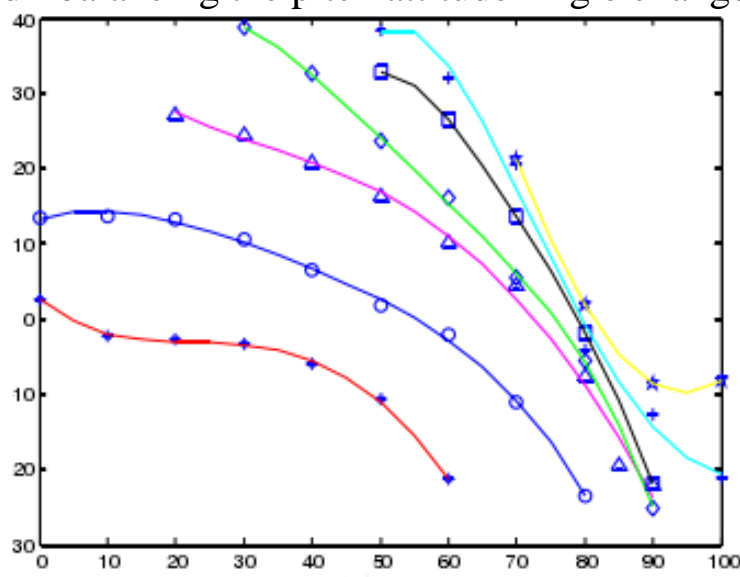

Figure 5. XV -15 balancing pitching Angle

Can be seen from the above five XV to 15 balancing attitude Angle in different dip Angle changing law is similar, are negative. In the dip Angle is less than $30^{\circ}$, the plane there is no minimum speed limit; In a fixed wing machine mode, the XV lower limit - 15 only flight speed, can be seen from the balancing attitude Angle and, in a fixed wing machine model, balancing attitude Angle in speed increase to $90 \mathrm{~m} / \mathrm{s}$ after the basic remained stable. In addition, analysis the change trend of balancing pitching Angle, it is recommended that the tilt rotor aircraft flying transition should be in the process of rotated nacelle gradually accelerated, to keep the aircraft pitch attitude Angle of the basic remain unchanged.

Transition from the corridor simulation it can be seen that the transition of the corridor to determine correlation with balancing attitude Angle is large, and the determination of transition route is based on balancing the attitude Angle to determine. As a result, the tilt rotor aircraft transition in the simulation research of corridor, balancing is a very important research method.

\section{Summary}

Corridor in transition, tilt rotor aircraft can be implemented smoothly switch between the working mode. In addition, all control research for transition section should be in transition to achieve within the corridor. Transition, therefore, the simulation research of corridor is not only a tilt rotor aircraft main technical points of the work mode switch, is also a transition section height, such as the premise of control research and validation.

\section{References}

[1] Gary B,Churchill Simulation of the XV-15 Tilt Rotor Research Aircraft. NASA Technical Memorandum 84222.

[2] Eric B.Carlson, Yiyuan Zhao, Robert T.N.Chen. Optimal Tiltrotor Runway Operations in One Engine Inoperative AIAA-99-3961:76 77.

[3] Renliang Chen. Control Strategy for Tilt-Rotor Aircraft Trimming in Steady Level Flight

[4] The 2nd International Basic Research Conference on Rotorcraft Technology, 2014.

[5] R.K.Mehra, R.K.Prasanth, S.Gopalaswamy.XV-15 Tiltrotor Flight Control System Design sing Model Predictive Control.IEEE, 1998.

[6] A. Okan,O. Tekinalp,M.S. Kavasaoglu, etal. Flight mechanics analysis of a tilt-rotor uav. aiaa-99-4255,1999 